\title{
BMJ Open Differences in access to Emergency Paediatric Intensive Care and care during Transport (DEPICT): study protocol for a mixed methods study
}

\author{
Padmanabhan Ramnarayan, ${ }^{\circ 1}$ Ruth Evans, ${ }^{\circ} 2$ Elizabeth S Draper, ${ }^{3}$ \\ Sarah E Seaton, ${ }^{3}$ Jo Wray, ${ }^{4}$ Stephen Morris, ${ }^{5}$ Christina Pagel, ${ }^{6}$ DEPICT Study \\ Investigators
}

To cite: Ramnarayan P, Evans R, Draper ES, et al. Differences in access to Emergency Paediatric Intensive Care and care during Transport (DEPICT): study protocol for a mixed methods study. BMJ Open 2019;9:e028000. doi:10.1136/ bmjopen-2018-028000

- Pre-publication history and additional material is published online only. To view please visit the journal online (http://dx.doi org/10.1136/bmjopen-2018028000).

Received 19 November 2018 Revised 4 June 2019 Accepted 6 June 2019
Check for updates

(C) Author(s) (or their employer(s)) 2019. Re-use permitted under CC BY-NC. No commercial re-use. See rights and permissions. Published by BMJ.

For numbered affiliations see end of article.

Correspondence to

Dr Padmanabhan Ramnarayan; p.ramnarayan@gosh.nhs.uk

\section{ABSTRACT}

Introduction Following centralisation of UK paediatric intensive care, specialist retrieval teams were established who travel to general hospitals to stabilise and transport sick children to regional paediatric intensive care units (PICUs). There is national variation among these PICU retrieval teams (PICRTs) in terms of how quickly they reach the patient's bedside and in the care provided during transport. The impact of these variations on clinical outcomes and the experience of stakeholders (patients, families and healthcare staff) is however unknown. The primary objective of this study is to address this evidence gap.

Methods and analysis This mixed-methods project involves the following: (1) retrospective analysis of linked data from routine clinical audits (2014-2016) to assess the impact of service variations on 30-day mortality and other secondary clinical outcomes; (2) a prospective questionnaire study conducted at 24 PICUs and 9 associated PICRTs in England and Wales over a 12-month period in 2018 to collect experience data from parents of transported children as well as qualitative analysis of in-depth interviews with a purposive sample of patients, parents and staff to assess the impact of service variations on patient/family experience; (3) health economic evaluation analysing transport service costs (and other associated costs) against lives saved and longer term measurements of quality of life at 12 months in transported children and (4) mathematical modelling evaluating the costs and potential impact of different service configurations. A final work stream involves a series of stakeholder workshops to synthesise study findings and generate recommendations.

Ethics and dissemination The study has been reviewed and approved by the Health Research Authority, ref: 218569 . Study results will be actively disseminated through peer-reviewed journals, conference presentations, social media, print and broadcast media, the internet and stakeholder workshops.

\section{INTRODUCTION}

Centralisation of specialist paediatric services in the UK has led to the establishment of
Strengths and limitations of this study

- This is the largest national mixed-methods multiple work stream study conducted in paediatric intensive care transport.

- The study will synthesise findings from four work streams to explore the impact of timeliness of intensive care transport on clinical outcomes and family experience.

- Extensive input from patients and service users, integrated health economic evaluation and novel mathematical modelling will provide evidence to support future service models.

- The study findings have the potential to change future national standards, guidelines and information resources for patients and families.

- Our study limitations include the lack of a single validated risk adjustment measure for the data analysis work stream and the potential for low return rate of parent questionnaires in work stream B.

regional paediatric intensive care units (PICUs) and paediatric intensive care retrieval teams (PICRTs), mobile intensive care teams that travel to general hospitals to initiate intensive care and transport critically ill children to PICUs. ${ }^{12}$ The use of PICRTs (rather than non-specialist teams) for the inter-hospital transport of critically ill children improves the odds of survival by $42 \%$; the majority $(\sim 85 \%)$ of such transports are currently performed by PICRTs with the remainder being performed by non-PICU teams. ${ }^{3}$

Retrieval services for children have evolved ad hoc over the past two decades, and currently differ in terms of how the service is organised and care provided during transport, in particular, seniority of the transport team leader (consultant, junior doctor or advanced nurse practitioner), critical care interventions performed (eg, intubation 
or central venous catheterisation) and the frequency of critical incidents (eg, accidental extubation) during transport. ${ }^{4}$ PICRTs also vary in the time taken to reach the patient from referral acceptance (median 1-4hours, although up to 24 hours during the winter surge in demand), impacting on how soon a critically ill child can expect to start receiving specialist paediatric intensive care (PIC) ${ }^{5}$ National quality standards recommending that PICRTs should reach the patient within 3 hours of referral acceptance are based on expert opinion due to a dearth of scientific evidence regarding the impact of delayed arrival of the PICRT on patient outcomes. In addition, there has been little systematic research into how the patient's experience is influenced by timeliness of access to PICRTs, and the quality of care delivered by PICRTs, at what is a particularly vulnerable period in critically ill children's (and their families') lives. Evidence of psychological trauma can be found long after acute transfer and admission to PICU, ${ }^{67}$ so exploring the impact of service variation on patients' and their families' experience may provide an opportunity to intervene and ameliorate an acutely difficult experience.

Transported children represent one-third of all UK PICU admissions (and one-half of all emergency admissions), yet have poorer outcomes than planned admissions or emergency admissions from within the same hospital where the PICU is located. ${ }^{3}$ It is not clear whether this is solely because transported children are much sicker, or whether other factors such as the timeliness of access to PICU expertise and care delivered by PICRTs prior to PICU admission have an additional influence on clinical outcome. Understanding the impact of variations in PICRT services may help to understand why outcomes in this group are worse and how to improve them.

This paper outlines the protocol of a national mixedmethods study that primarily aims to explore how clinical outcomes of critically ill children transported to paediatric intensive care (PIC) are affected by variations in both timeliness of access to PIC and the care provided by PIC retrieval teams during stabilisation and transport. Secondary objectives include understanding the experience of the transport of critically ill children from the perspectives of different stakeholders (patients and families, and healthcare staff from referring hospitals, PICRTs and PICUs); health economic analysis to explore the relative cost-effectiveness of current PICRTs and mathematical modelling to evaluate whether alternative models of PICRT service delivery can improve clinical and cost effectiveness.

\section{METHODS AND ANALYSIS}

\section{Study design}

As shown in figure 1, Differences in access to Emergency Paediatric Intensive Care and care during Transport (DEPICT) is a mixed-methods study with multiple work streams: (1) work stream A (retrospective quantitative analysis of linked data from routine clinical audit and administrative sources); (2) work stream B (prospective observational study involving administration of questionnaires to parents of transported children as well as parent, patient and staff interviews); (3) work stream C (health economic evaluation); (4) work stream D (mathematical modelling) and (5) work stream E (synthesis of findings from the four work streams and stakeholder workshops to refine study findings).

\section{Setting}

The study will involve critically ill children and young people (CYP) (before their 16th birthday) transported for emergency admission to 24 PICUs (representing 21 National Health Service (NHS) Trusts) by 9 PICRTs in England and Wales.

\section{Recruitment and analysis plan by work stream Work stream A}

The objectives of this work stream are to study the association between (1) timeliness of access to a PICRT and clinical outcomes and (2) care delivered by PICRTs and clinical outcomes.

\section{Study dataset}

We will create a study dataset by linking four different sources of routinely collected audit and administrative data: (1) Paediatric Intensive Care Audit Network (PICANet) comprising clinical information regarding the case mix, resource use and outcome of $\sim 15000$ children transported to PICUs between 1 January 2014 and 31 December 2016; (2) Intensive Care National Audit and Research Centre (ICNARC) Case Mix Programme (CMP) consisting of clinical information on children admitted to adult critical care units prior to paediatric transport; (3) Hospital Episode Statistics (HES) comprising administrative and clinical data on acute hospital attendance and admissions from English hospitals as well as similar data from the NHS Wales Informatics Service and (4) Office of National Statistics (ONS) mortality data.

\section{Data linkage}

PICANet and ICNARC will provide patient-level identifiable data (NHS number, sex, postcode and date of birth) about emergency paediatric admissions to intensive care to NHS Digital (https://digital.nhs.uk/) for data linkage, including a pseudo-anonymised identifier. NHS number will be the primary identifier to allow matching between data sources, and this has high levels of completeness in both the PICANet $(98 \%)$ and CMP (97\%) data. NHS Digital will merge these data sources and link them with HES and ONS. Identifiable data will be removed by NHS Digital before providing the linked data to the statistical team at the University of Leicester. Additional clinical and demographic data will be provided from PICANet and ICNARC to the statistical team using the study-specific patient-level pseudo-anonymised identifier to link the data. The data flow to and from NHS Digital is described in figure 2. Additional information for Wales will be requested and will follow a similar data flow to that in figure 2, although linkage will only take place on NHS number. 


\section{DEPICT STUDY}

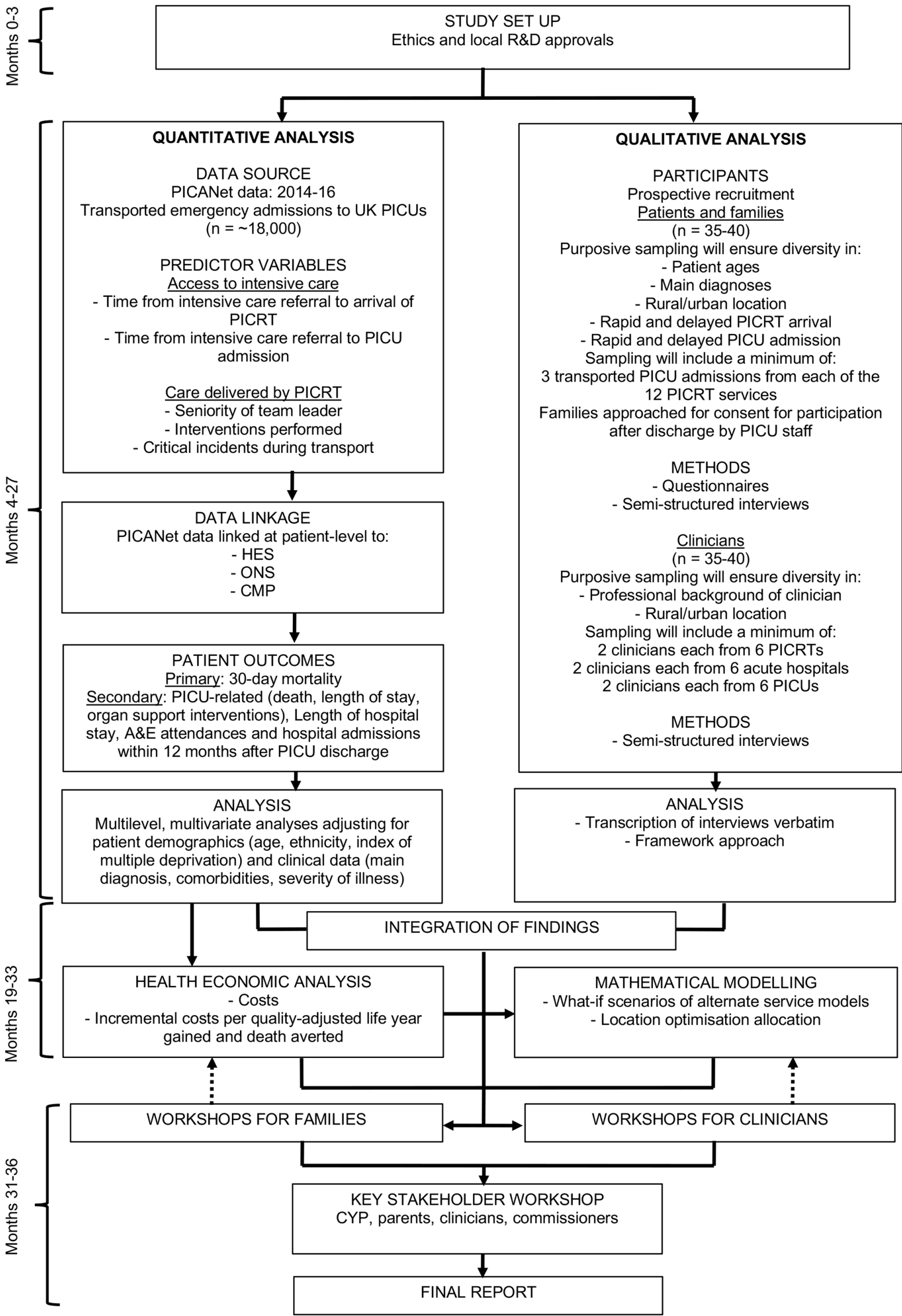

Ethics and local R\&D approvals

Figure 1 Study flow diagram illustrating the study work streams and their relationship. PICANet, Paediatric Intensive Care Audit Network; PICRT, paediatric intensive care retrieval team; PICU, paediatric intensive care unit. 
FLOW OF DATA IN THE DATA LINKAGE PART OF THE DEPICT STUDY

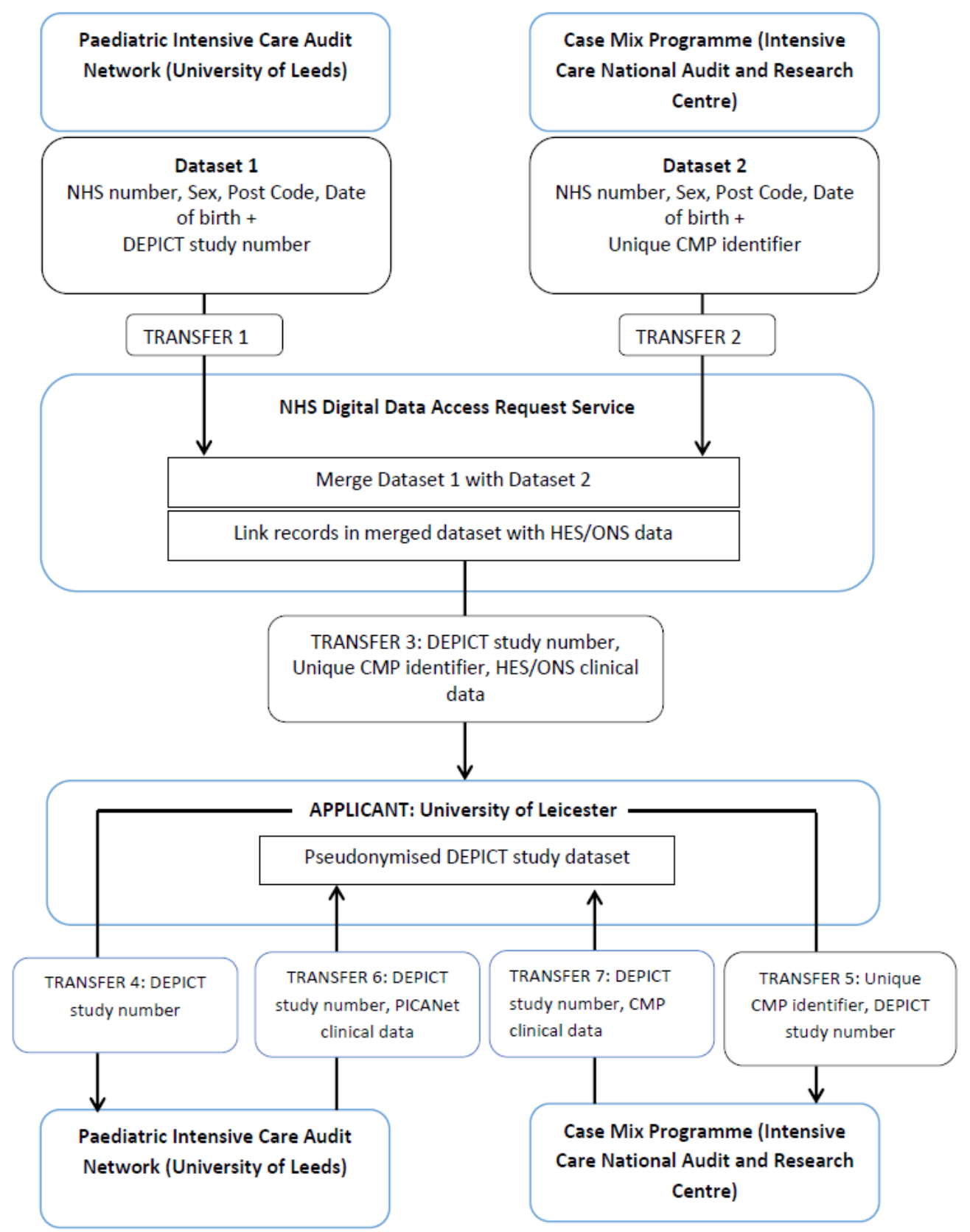

Figure 2 Flow of data to and from NHS Digital for the data linkage work stream of the DEPICT Study. CMP, Case Mix Programme; DEPICT, Differences in access to Emergency Paediatric Intensive Care and care during Transport; HES, Hospital Episode Statistics ; NHS, National Health Service; ONS, Office of National Statistics; PICANet, Paediatric Intensive Care Audit Network.

\section{Study population}

All transports of critically ill children (before their 16th birthday) to a PICU in England and Wales from 1 January 2014to 31 December 2016 will be included. Exclusions will be made for no match between PICANet transport and PICU admission data, and transports by neonatal or local/non-specialist teams.

\section{Outcomes}

The primary outcome will be mortality within 30 days following PICU admission (the first admission in case of multiple PICU admissions). Secondary outcomes, selected by clinical members of the research team to complement the primary outcome, will include (1) mortality at PICU discharge, 90 days and 1 year following (first) PICU admission; (2) number of PICU admissions during study period and time to readmission (if applicable); (3) length of stay in PICU for each PICU admission; (4) resource use in PICU (number of days of invasive ventilation, vasoactive agent therapy, renal replacement therapy and extra-corporeal life support); (5) length of hospital stay linked to the (first) PICU admission; (6) number of emergency department attendances in the 12 months following 
discharge from PICU and (7) hospital resource use in the 12 months following PICU discharge.

\section{Statistical analysis}

The full statistical analysis plan is available on the study website (https://depict-study.org.uk/study-documents). Summary information will be provided including the following:

- Characteristics of the transports undertaken, for example, grade of team leader, n (\%).

- Interventions during or prior to transport conducted by the retrieval team, n (\%)

For example, intubation, central venous catheter insertion, adverse events during transport (medical: eg, accidental extubation, or vehicular: eg, road traffic accident).

- Primary diagnosis reason from evidence available at the time of admission, $\mathrm{n}(\%)$

For example, respiratory, neurological, cardiovascular, infection, endocrine/metabolic, haematology/ oncology, trauma, other.

- Reason for admission, as identified during admission, $\mathrm{n}(\%)$

For example, bronchiolitis, seizure disorder, asthma, croup, postoperative, diabetic ketoacidosis.

- Paediatric Index of Mortality (PIM)-2/PIM-3 score, median (range) and grouped as $<1,1$ to $<5$; 5 to $<15$, 15 to $<30,30-100$.
- Mortality at 30 days, 90 days and 1 year after first PICU admission.

- Length of stay, median (range).

- Daily interventions in the PICU (eg, ventilation) and median days treatment received (range).

\section{Missing or implausible data}

At this stage, we are unaware of the level of missing data which will be encountered; however, data quality and completeness of the PICANet and CMP data are believed to be high. Missing data will still occur and when levels of missing data are high $(>10 \%)$ for key variables, reasons for this will be investigated, for example, whether one particular transport team has a certain variable consistently missing. If we believe data are missing at random, we will investigate use of multiple imputation. We will develop robust proposals for handling each variable as we become aware of the level of missingness and present these to the Study Management Group for approval before analysis is conducted.

\section{Formal statistical analysis}

Two main statistical models will form the basis of the primary analysis: see figure 3 for a graphical representation of the entire transport pathway. Model 1 will investigate the impact on mortality due to the timeliness of access to care for children requiring transport by a PICRT. PICANet collects data on all key time points within the transport including, time transport was agreed; time the

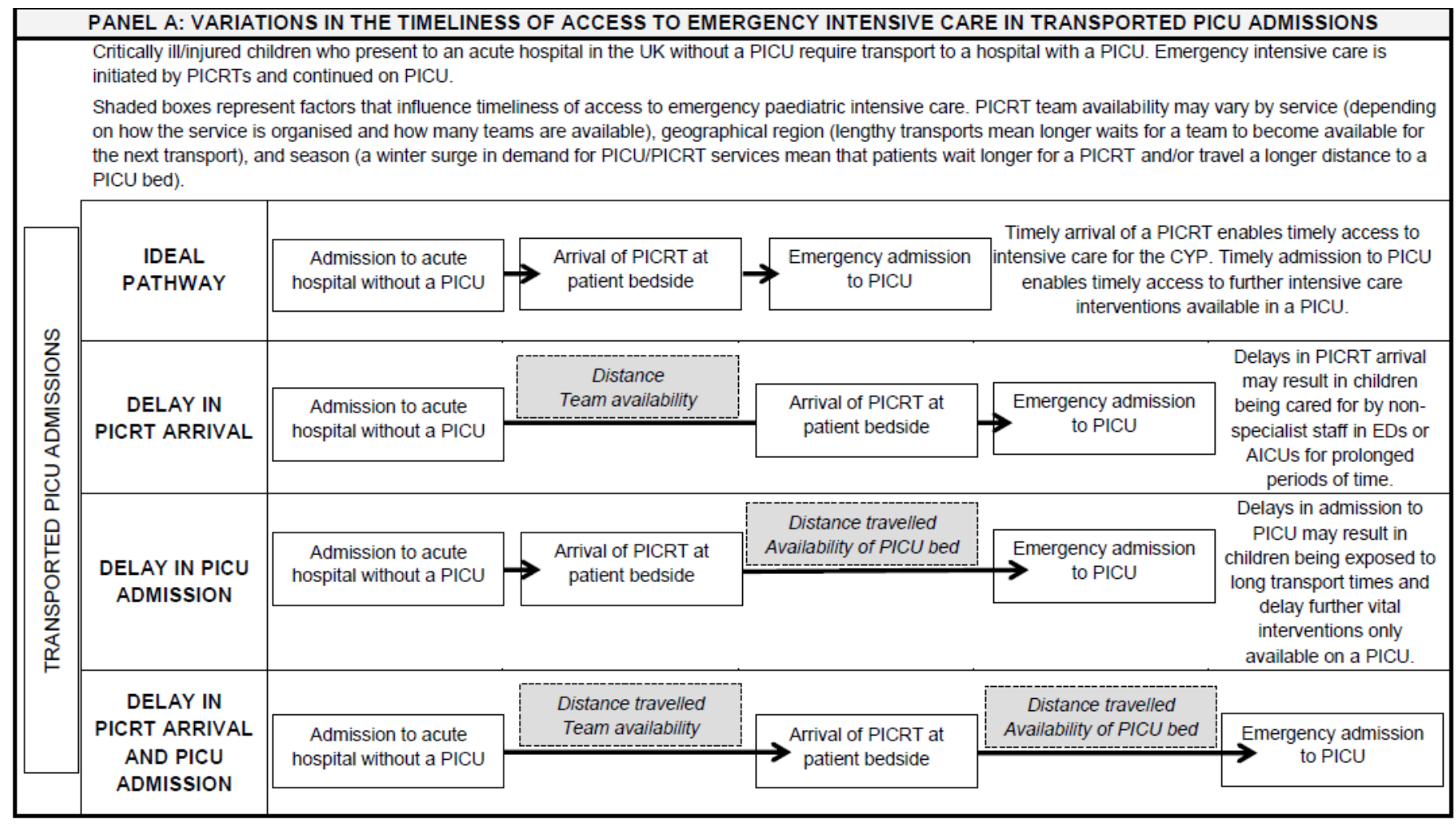

Figure 3 Graphical illustration of the reasons for delay in a PICRT reaching the patient's bedside at the referring hospital and the patient reaching the PICU. AICUs, adult intensive care units; CYP, children and young people; EDs, emergency departments; PICRT, paediatric intensive care unit retrieval team; PICU, paediatric intensive care unit. 
Table 1 List of potential confounders for consideration in each of the statistical models

\begin{tabular}{ll}
\hline Variable & Data source \\
\hline Age & PICANet and CMP \\
Sex & PICANet and CMP \\
\hline Ethnicity & HES, PICANet and CMP \\
$\begin{array}{l}\text { Pre-existing comorbidities (or a proxy, eg, time in hospital in } \\
\text { the previous year) }\end{array}$ & HES, PICANet and CMP \\
$\begin{array}{l}\text { Deprivation (IMD score) } \\
\text { Diagnosis }\end{array}$ & PICANet and CMP (via postcodes or LSOA) \\
$\begin{array}{l}\text { Severity of illness (PIM-2/PIM-3 score and individual variables } \\
\text { from first contact with transport team, not from referral) }\end{array}$ & PICANet, CMP and HES \\
Ventilated at referral & PICANet \\
\hline Previous admission to intensive care & PICANet and CMP \\
Volume of referring hospital & HES or RCPCH data \\
Interventions prior to arrival of the transport team & PICANet \\
Referral location (A\&E, HDU, theatre, ward etc.) & PICANet \\
Season (winter defined as Nov to Feb) & PICANet \\
In hours versus out of hours (to include evenings and & PICANet \\
weekends) & \\
\hline Interventions conducted when retrieval team arrives & PICANet \\
\hline Grade of team leader & PICANet \\
\hline
\end{tabular}

CMP, Case Mix Programme; HDU, High Dependency Unit; HES, Hospital Episode Statistics; IMD, Index of Multiple Deprivation; LSOA, lower super output area; PICANet, Paediatric Intensive Care Audit Network; PIM, Paediatric Index of Mortality; RCPCH, Royal College of Paediatrics and Child Health.

transport team set off; time the team arrived at the district general hospital and time the team arrived at the PICU. Model 2 will investigate the care delivered by the transport team on the way to the PICU and the impact this has on mortality.

Model building for both analyses will follow these steps:

1. Selection of key confounders via statistical and clinical discussions (see table 1).

2. Drawing of a directed acyclic graph (DAG) to represent the model to be fitted ${ }^{8}$ and to ensure minimal adjustments are used. This DAG will be approved by the Study Management Group and published in papers related to its use.

3. Formal regression model will be fitted (eg, logistic model with mortality as the outcome to compare whether, after adjustment, the time to arrive at the patient's bedside impacts on the odds of mortality). Different approaches to modelling covariates will be considered including fractional polynomials and splines if their use provides a difference of at least 4 in the Akaike Information Criterion (AIC) between the models. The AIC allows comparison between multiple statistical models to identify the most appropriate model to use.

4. Model checking will be conducted including, HosmerLemeshow test, c-statistic and Briers score (or equivalent model checking methods for other approaches).
Stability of model estimates will be assessed via bootstrapping.

Sensitivity analyses will be conducted to investigate any assumptions, including missing data and unobserved confounding (using an instrumental variables approach), ${ }^{9}$ and the robustness of the data.

There may be children with more than one transport recorded in the data. For these children, we will either conduct the analysis on their most recent transport, or cluster on patient identifier to adjust the standard errors or use a random effect within a generalised linear mixed model. Again, this will form part of our sensitivity analyses.

The analysis will be repeated for secondary outcomes, using the following statistical approaches:

- Length of stay: survival analysis approaches including competing risks.

- Resource use in PICU and hospital resource use following PICU discharge: negative binomial models (to account for the fact that many children will have no resource use for certain interventions).

- $A \mathcal{E} E$ attendances: logistic regression (re-admitted to A\&E: yes/no) or Poisson regression (number of re-admissions).

All assumptions for methods used will be investigated to ensure our approaches are robust, for example, we will check for overdispersion when using negative binomial models. All analyses will consider the potential clustering 
within the data, including the transport teams, and some patients have multiple transports. Where appropriate, multilevel models or methods to account for clustering (eg, robust standard errors) will be used.

\section{Sample size}

We anticipate a sample size of 10000 to 15000 transports as seen in previous years of transport data collected by PICANet (https://www.picanet.org.uk/annual-reportingand-publications/). A formal prospective sample size calculation was not possible, as in reality, we will use all available data and ensure our level of adjustment is reasonable compared with the number of events to ensure we do not over-fit the model.

\section{Reporting conventions}

Adjusted odds ratios, risk ratios or other coefficients will be reported to one decimal place with $95 \%$ CIs. P values will not be presented and consideration will be given to both statistical and clinical significance. Small numbers will be suppressed in line with HES guidance (http:// docs.adrn.ac.uk/888040/mrdoc/pdf/888040_hes-analyguide-apr13.pdf).

\section{Work stream B}

The objectives of this work stream are to explore (1) parents', and where feasible, children's, experiences of emergency transport to PICU and (2) clinicians' and service managers' (or commissioners') perspectives of PICU transport and its impact on care provided to critically ill CYP, and the wider impact on other patients and services.

\section{Recruitment of transported CYP and their parents}

Parents of critically ill CYP admitted to PICU over a 12 -month period from January 2018 will be recruited. All emergency admissions to participating PICUs over this period will be screened for eligibility; CYP transported by PICRTs (or other transport teams) will be eligible.

Parents/guardians of eligible patients will be approached by clinical/research staff while their child is on PICU to discuss the study and obtain written informed consent for (1) completion of a questionnaire relating to the experience of PIC transport; (2) potential contact by a researcher for participation in an interview 3-6 months after PICU admission; (3) contact by researchers for completion of a child quality of life questionnaire (Peds$\mathrm{QL}$ ), generic quality of life questionnaire (HUI-2) and asked details of use of health services and other family costs 12 months after PICU admission and (4) the use of patient identifiers to extract PICANet data relating to the child's transport episode.

Relevant information sheets for the study will be available in English as well as six other languages (Bengali, Gujarati, Punjabi, Urdu, Polish and Welsh-chosen based on the most frequently spoken languages in England and Wales from 2011 census data) to support the process of informed consent. Separate procedures have been devised to recruit parents of CYP who die in the PICU.
These procedures involve sending a personalised letter from the site principal investigator and information sheets and consent form approximately 6-8 weeks after the child's death.

\section{Questionnaire study}

Parents who agree to participate will be given a paper questionnaire, developed specifically for this study from existing questionnaires, review of relevant literature and using the experience of the study team, steering group and parent representatives to further inform the format and content. Parents will also be able to complete the questionnaire electronically, including a speak-aloud option to facilitate completion for participants with low literacy levels. Where appropriate, two copies of the questionnaire will be offered to families recognising that parents may have different perspectives and capturing both would enrich the description of family experiences. The questionnaire uses a mixture of rating scales, tick boxes and free text boxes to collect parental responses to specific questions regarding their experience before, during and after their child's transport to PICU and includes questions related to arrival of the PICRT, information provided about the transport process, expectations about the transport team and whether these were met and whether the family were able to accompany the child in the transport. Demographic questions will be asked but no identifiable information will be recorded. The questionnaire was piloted with several families at two PICUs to check coherence, clarity and acceptability. If consent is provided, questionnaire responses will be linked with PICANet data on that specific patient transport to provide additional information for analysis (eg, exact time of arrival at patient's bedside).

\section{Interview study}

We will interview a purposive sample of parents, CYP and healthcare staff. Interview topic guides for staff and parents/guardians have been provided as online supplementary file 1 .

\section{Parents}

Eligible parents will be invited 3-6 months after their child's admission to PICU if they have completed the transport questionnaire and consented for interviews. Use of a sampling matrix will ensure diversity in terms of child's age, diagnosis, distance from referring hospital to PICU, previous use of PICRTs and whether parents travelled with the child in the ambulance. We anticipate recruiting 50 parents, which should be a sufficiently large number to capture the range of parents' experiences across the different models of care and to reach data saturation, balanced with resource implications and feasibility of collecting and analysing large amounts of interview data. Interviews will be completed face-to-face unless a preference is expressed for telephone or Skype, and written consent obtained/recorded. Interviews will be recorded and transcribed verbatim. Interpreters 
will be arranged if necessary to support participation of non-English-speaking families. The interview topic guide will encourage families to talk about their experiences at different stages of the transport journey with a specific focus on talking about what went well, what worked less well and what an optimum service would look like.

\section{Children and young people}

CYP aged 5-16 years, who can remember some of their transport journey and are able to provide age-appropriate assent for their participation, will be eligible. We aim to create a diverse sample similar to that described for parent interviews but will employ a more flexible recruitment strategy recognising the challenges of recruiting eligible CYP. We will use a range of creative methods to engage CYP including draw and write, ${ }^{10}$ talking mats ${ }^{11}$ and third-person craft activities. Sessions will be audiorecorded, photographs taken of any creative outputs and notes taken immediately after the session about aspects such as CYP's engagement and their ability to recall the experience and the impact of revisiting the time of their transport to PICU.

\section{Clinicians and service managers}

We will recruit 35-40 health professionals from PICRTs, PICUs and a representative sample of acute general hospitals; we aim to create a diverse sample in terms of professional background, experience, place of work and experience of emergency transfers. Participants will be recruited via an email advert circulated by local site principal investigators. Interested staff will be encouraged to contact the study researchers and will be sent an information sheet and consent form to complete before participation. Interviews will focus on asking participants to describe a transfer that went well, one that went less well, to discuss the wider impact of the PIC retrieval service on the care of other children and services and to describe what they feel an optimal service would look like. Initial piloting with three to four clinicians will enable further refinement of the topic guide.

\section{Data analysis}

Based on published response rates from postal questionnaires in the area of paediatric critical care, we estimate a return rate of 25\% (anticipated 800-1000 returned questionnaires) but we will work with PICU research teams to maximise return rate to improve the generalisability of our findings. Descriptive statistics will be used to describe and compare different PICRT models and to examine key variables. We will assess the impact of missing data by repeating analyses with and without participants with missing data. We will assess whether missing data is random or systematic and report decisions made about missing data. Interview transcripts will be entered verbatim into NVIVO, a qualitative data analysis programme. The Framework approach will be used to enable thematic analysis of described experiences. Framework facilitates rigorous and transparent data management, involving five distinct stages: familiarisation, identifying a thematic framework, indexing, charting, mapping and interpretation. ${ }^{12}$ Data will be compared within cases (people and PICRTs) and across cases, and this will allow comparisons of experience between different PICRTs and between different models of care.

\section{Work stream C}

The objective of this work stream is to evaluate the cost-effectiveness of PICRT provision for critically ill children, comparing different services models that are currently in use, varying in terms of team composition, availability of interventions in transit and timeliness of access to the PICU. This will use the datasets created in work streams A and B, supplemented with NHS cost data.

The primary outcome measure will be number of lives saved in each strategy; the secondary outcome will be quality-adjusted life years (QALYs). For costs, an NHS and Personal Social Services (PSS) perspective will be adopted in the base case, and a societal perspective will be adopted in sensitivity analysis. Costs and outcomes will be evaluated using several time horizons, for example, up to 30 days and 1 year following PICU admission, and lifetime.

\section{Measuring costs}

Costs will include PICRT transport and broader NHS/ PSS and societal costs. A detailed cost analysis of transport by the PICRT will be carried out based on travel time, team composition, interventions performed and management of critical incidents, based on PICANet data from work stream A.

The main NHS costs will include time spent in the PICU, time spent on different wards in the hospital after discharge from the PICU, and hospital resource use in the 12 months following PICU discharge (admissions, emergency department visits, outpatient visits) obtained from PICANet data from work stream A. Unit costs for each of these items will be obtained from provider trusts and published sources, producing a patient-level cost dataset for all patients in work stream A. Primary care costs will be obtained from 12-month follow-up questionnaires (work stream B), which will include accommodation, travel, food and drink costs as well as time off work, and combined with NHS costs to compute societal costs. Mean primary care and family costs will be computed for participants in workstream B grouped by primary and secondary outcomes and confounding variables used in in workstream A; mean group costs will then be mapped onto the patient-level data in workstream A. Costs beyond 1 year will be modelled from published sources based on systematic literature searches of the Cost-Effectiveness Analysis (CEA) Registry at Tufts University, the NHS Economic Evaluations Database, the Health Technology Assessment Database, the Research Papers in Economics Database and PubMed. ${ }^{13}$ All costs will be reported in constant prices, inflated where necessary using published inflation indices. ${ }^{14}$ 


\section{Measuring outcomes}

Mortality up to 1 year will be measured directly in the study (work stream A). Quality of life will be assessed at 12 months and combined with data on survival to compute QALYs. Measuring health-related quality of life suitable for estimating QALYs in our population of mainly $<5$-year-old children is challenging. We therefore propose to use two methods: HUI-2 measured via proxy assessment by the parent and the PedsQL mapped to EQ-5D scores, both obtained from the follow-up questionnaires in workstream B. ${ }^{15-17}$ Mean utility scores by primary and secondary outcomes and confounding variables used in work stream A will be calculated for patients completing the questionnaire in work stream $B$ grouped and then mapped onto the patient-level data in work stream A, to produce a patient-level QALY dataset for all patients in workstream A. Long-run survival and utility data suitable for estimating lifetime QALYs will be obtained from published sources including the CEA Registry at Tufts University, which contains a searchable database for utility weights by, for example, diagnosis; the NHS Economic Evaluations Database and systematic searches of the wider literature for quality of life information suitable for estimating QALYs, for example, using PubMed.

\section{Analysis}

We will produce a patient-level dataset of costs and outcomes for every patient in work stream A. We will use this to analyse the costs and outcomes associated with different PICRT models using regression analysis. ${ }^{18}$ We will also follow current guidance on conduct of economic evaluations using observational data to assess the main assumptions for addressing selection bias in the statistical models implemented, including assessing the "no unobserved confounding' assumption, overlap of baseline covariates between patients in the different PICRT models and the specification of the regression models. ${ }^{19}$ The regression analyses will estimate lives saved, differences in costs and QALYs gained between different PICRT models. Cost-effectiveness will be measured using incremental net monetary benefits calculated at different values of the willingness to pay to avoid one death or gain one QALY. We will undertake extensive sensitivity analysis to evaluate the impact of uncertainty in our analysis.

We will follow current guidance for methods of technology appraisal to present and report the results of the economic analysis. ${ }^{20}$ We will estimate the budget impact if each PICRT model were to be rolled out nationally based on projections of need for PICRT services.

\section{Work stream D}

Work stream D will use mathematical modelling and location allocation optimisation methods to explore whether alternative models of service delivery for PICU/PICRT services can improve clinical outcomes without increasing overall cost. This work will build directly on the quantitative analysis (work stream A) and the health economic evaluation (work stream $\mathrm{C}$ ), but will also incorporate insights gained from the qualitative work (work stream B).

We note that these methods are not statistical modelling - they are a form of optimisation modelling where the aim is to optimise a given decision-dependent goal (eg, making journey times as short as possible by changing the location of retrieval teams) subject to various constraints (eg, maximum number of retrieval team locations and minimum level of demand per location). In simple optimisation models there are exact solutions. For more complex problems that are computationally complex, heuristic algorithms are used to obtain a solution. There is no formal statistical validation of these modelsinstead, we will carry out sensitivity analysis to understand the impact of small changes to the objective function, its coefficients and constraints on the resulting output (location and numbers of PICRTs). This will provide an assessment of the robustness of the modelling approach to this problem.

In particular, we will use mathematical modelling and optimisation methods to explore the potential impact on outcome of different possible models of service that could impact the identified drivers of better outcomes. Possible alternative models could include the following:

More transport services (to reduce distance) (either with same number of teams or more teams).

The same number of transport services with more teams (to increase team availability).

Seasonal allocations of teams (to plan for the winter surge).

Changes to team composition.

We will also use location-allocation optimisation to investigate, for a given number of transport services and teams and a set of possible locations, where PICRTs should be based to minimise travel time to the local hospitals they serve and to the receiving PICUs. The constraints on the numbers of services, numbers of teams and possible locations will be defined through conversations with the PICRT services and commissioners.

Potential service models will also take into account the views of parents and staff, in particular where there are options that are clearly preferred and would not negatively affect the child's eventual outcome.

Any proposed service models will be further re-examined in light of the feedback from stakeholders (parents and families, PICUs, PICRTs and local hospital clinicians) from the workshops (work stream E). We will also combine the results of this modelling strand with the cost-effectiveness analysis, by estimating the costs associated with different service configurations and the anticipated cost of outcomes associated with that configuration (for instance, if longer journey times lead to poorer clinical outcomes). It is possible that time to bedside is only one important factor; we will explore solutions to the model that also seek to minimise time from bedside to receiving PICU and time from receiving PICU back to base (which will affect team availability). We will account for stochastic journey and mobilisation 


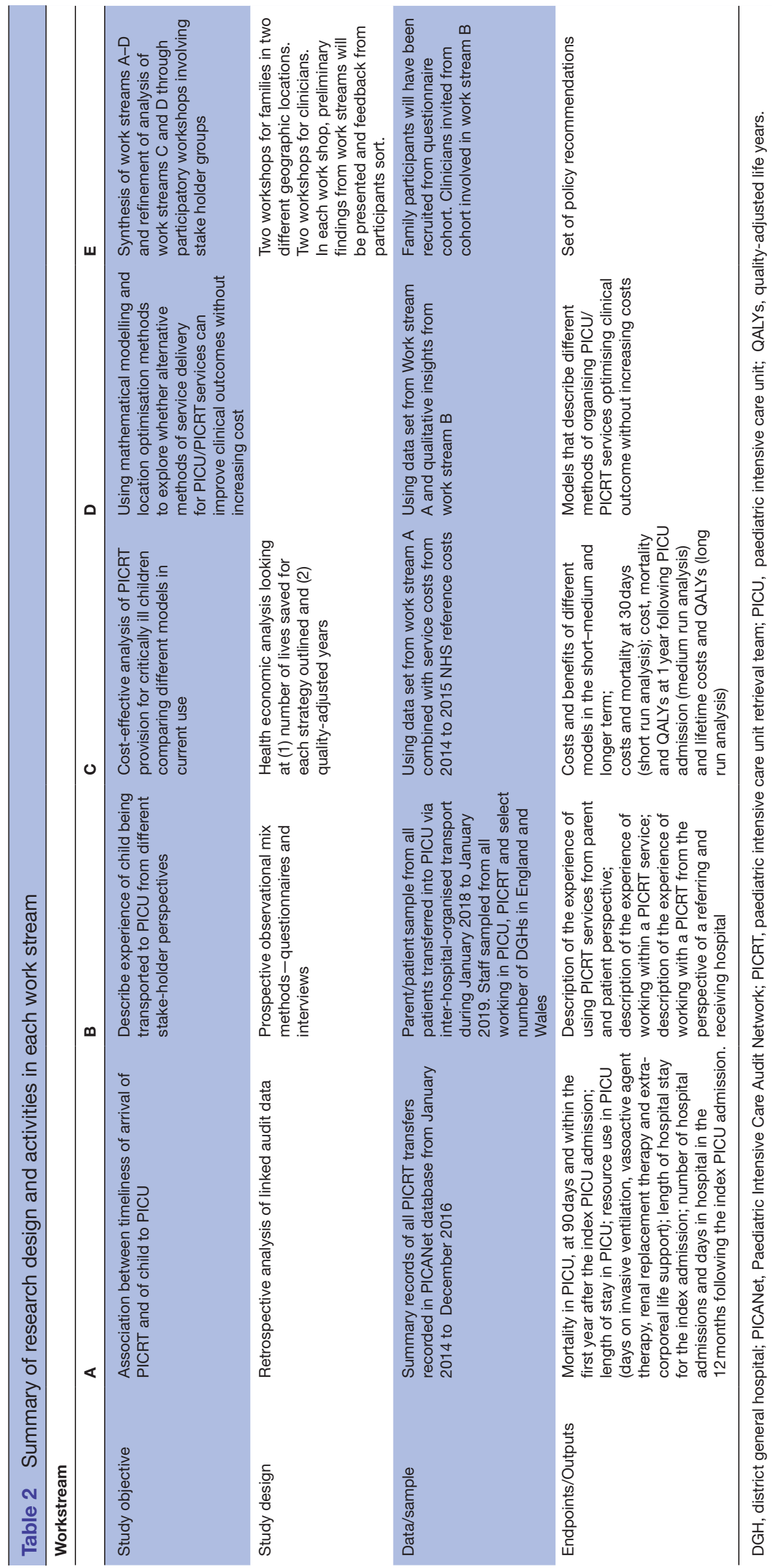


times and explore how best to incorporate seasonal demand.

Deciding where best to locate PICRTs also needs to incorporate decisions as to the number of teams available for each retrieval service. Queueing theory such as that deployed by Pagel in previous work for the Children's Acute Transport Service retrieval team will be used to determine the relationship between referral rates, the number of retrieval teams that a service has and the expected time to a team being available to despatch. We will also investigate whether the application of hypercube queuing models as first developed by Larson to optimise the location and number of emergency service vehicles could be adapted for the PICRT context. ${ }^{21}$ Developed models for this stream are likely to need heuristic solution algorithms, and the locations and allocations suggested by the previous analyses will then be used as starting solutions in a heuristic search to give locations and team numbers that minimise the total number of teams required to meet a particular service standard or to give the best service that uses a fixed number of teams.

Finally, we will explore the scope for extending the analysis to identify PICRT team sizes and locations that, as a set, maximise expected survival based on patterns of forecasted demand and a causal interpretation of the statistical associations identified between factors influenced by location of PICRTs and clinical outcome from work stream A.

\section{Work stream $\mathrm{E}$}

We will synthesise the findings from the work streams A to $\mathrm{D}$ and organise stakeholder workshops (see summary of work streams-table 2). We will arrange two workshops for families as well as for clinicians in different geographical locations to present the preliminary results of the work streams and to collect their feedback, which will be instrumental in informing any final analyses required within the health economic evaluation and the mathematical modelling work streams. A final key stakeholder workshop (families, clinicians, commissioners) will be held to present and synthesise results from each work stream. Workshop participants will be asked to consent to the sessions being audiorecorded, with assurance that no individual contribution will be directly attributable to them in any outputs.

\section{Patient and public involvement}

Parents of sick children retrieved to UK PICUs have regularly highlighted how the care provided during transport crucially influences their child's outcome and the family's experience. Two parent representatives form part of the study management group to inform the study design and conduct, especially related to data collection with parents, and help develop appropriate parent information resources. There are two additional patient representatives as part of the study steering committee, responsible for overall supervision of the research. The results of the study will be presented to key stakeholders and user groups will be closely involved with dissemination of study findings.

\section{Ethics and dissemination}

We have approval from the Health Research Authority, the National Research Ethics Service-London Riverside Committee (ref: 17/LO/1267) and the Confidentiality Advisory Group for the DEPICT study. As part of work stream A, no patient-identifiable data will be made available to the DEPICT study team by NHS Digital after linkage. In work stream B, families will be consented for the questionnaire study at a suitable time after PICU admission as judged by the clinical team. We will also have established procedures to manage the sensitive issues surrounding the interviews of parents/children, including the use of staff with previous experience of interviewing children/families on sensitive issues, clear policies for support for families and to stop interviews at any time if parents wish to do so.

Study findings will be disseminated widely using a comprehensive dissemination strategy, which will include the use of various forms of media to reach a diverse range of clinicians, managers and the public at the local, national and international levels. Academic media (peer-reviewed journal articles, conference presentations), the internet (www.depict-study.org.uk), social media (Twitter: @DEPICT_Study), print and broadcast media (newspaper, television and radio), electronic mail (study findings to clinicians and other stakeholders) and stakeholder engagement activities (workshops, meetings) will be employed to disseminate study findings.

\section{Author affiliations}

${ }^{1}$ Children's Acute Transport Service, Great Ormond Street Hospital For Children NHS Trust, London, UK

${ }^{2}$ Centre for Outcomes and Experience Research in Children's Health, Illness and Disability (ORCHID), Great Ormond Street Hospital For Children NHS Foundation

Trust, London, UK

${ }^{3}$ Department of Health Sciences, University of Leicester, Leicester, UK ${ }^{4}$ Cardiorespiratory Division, Great Ormond Street Hospital for Children, London, UK

${ }^{5}$ Department of Applied Health Research, University College London, London, UK

${ }^{6}$ Clinical Operational Research Unit, University College London, London, UK

Collaborators The DEPICT Study Investigators are as follows: Eithne Polke, Paul Mouncey, Rachel Lundy, Matthew Entwistle (patient representative), Anna Pearce (patient representative), Patrick Davies, Fatemah Rajah, Will Marriage. Great Ormond Street Hospital: Samiran Ray, Lauran O'Neill, Eugenia Abaleke, Yvonne Leonard, Ana Luisa Tomas, Holly Belfield, Guilia Scarpa Birmingham Children's Hospital: Sanjay Revanna, Lauren Dowd, Julie Menzies, Helen Winmill Evelina London Hospital: Benedict Griffiths, Paul Wellman, Tabby Crae Manchester Children's Hospital: Dorthe Grainger, Claire Jennings, Rebecca Marshall, Rajesh Phatak. Sheffield Children's Hospital: Fatemah Rajah, Sue Lenthall, Esther Ludbrook, Anton Mayer Bristol Children's Hospital: Peter Davis, Katherine Baptiste, Sarah Mogan, Will Marriage Great North Children's Hospital, Newcastle: Aravind Kashyap, Christine Mackerness, Dominic Manning, Lynda Pittilla Nottingham Children's Hospital: Patrick Davies, Lindsay Crate, Kelly Sandhu Leicester Royal Infirmary and Glenfield Hospital: Peter Barry, Rekha Patel Southampton Children's Hospital: Gareth Jones, Nichola Etherington, Helen Gale, Christie Mellish. St Mary's Hospital: David Inwald, Sarah Darnell, Suzanne Lang, Ladan Ali King's College Hospital: Akash Deep, Aravind Anand, Dammy Adebayo, Mehak Bansal Addenbrookes Hospital: Nazima Pathan, Deborah White, Esther Daubney Royal Brompton Hospital: Angela Aramburo, Laura Gemar, Laura TousSampol, Helena Fernandes Sampaio. Alder Hey Children's Hospital: Nayan Shetty, Laura Walsh, Dawn Jones Royal Stoke Hospital: John Alexander, Emily Obhrai, Victoria Riches Leeds General Infirmary: Joanne Lumsden, 
Janet Clarke St George's Hospital: Nicholas Prince, Elena Maccacari, Joan Gomes Royal London Hospital: Naomi Edmonds, Bessie Crone, Nosheen Khalid, Hafiza Khatun, Tara. Murray Oxford John Radcliffe Hospital: Avishay Sarfatti, Nicola Howell Noah's Ark Children Hospital for Wales: Siva Oruganti, Rhian Thomas Turner, Rhiannydd Poynter, Susan Bowes.

Contributors PR is the chief investigator of the study and drafted the manuscript. ESD, JW, SM and CP are study co-applicants and work stream leads, and made significant contributions to the protocol. All DEPICT Study Investigators made significant contributions to the protocol during study design (EP, PM, RL, ME, AP, PD, FR and WM). SES is a study statistician and contributed to aspects of the protocol related to work stream $A$. $R E$ is a research associate and contributed to aspects of the protocol related to work stream B. All authors commented critically on the manuscript and read and approved the final manuscript.

Funding This work was supported by the National Institute of Health Research Health Services Delivery Research program (NIHR HSDR ref: 15/136/45). The study is supported by the National Institute for Health Research Biomedical Research Centre at Great Ormond Street Hospital for Children NHS Foundation Trust and UCL Great Ormond Street Institute of Child Health. The study sponsor is Great Ormond Street Hospital NHS Foundation Trust, Joint R\&D Office GOSH/ICH, London, UK (email: Research.Governance@gosh.nhs.uk).

Competing interests None declared.

Patient consent for publication Not required.

Provenance and peer review Not commissioned; externally peer reviewed.

Open access This is an open access article distributed in accordance with the Creative Commons Attribution Non Commercial (CC BY-NC 4.0) license, which permits others to distribute, remix, adapt, build upon this work non-commercially, and license their derivative works on different terms, provided the original work is properly cited, appropriate credit is given, any changes made indicated, and the use is non-commercial. See: http://creativecommons.org/licenses/by-nc/4.0/.

\section{REFERENCES}

1. Department of Health. Paediatric intensive care: A framework for the future. Wetherby: Department of Health, 1997.

2. Ramnarayan $P$, Polke $E$. The state of paediatric intensive care retrieval in Britain. Arch Dis Child 2012;97:145-9.

3. Ramnarayan P, Thiru K, Parslow RC, et al. Effect of specialist retrieval teams on outcomes in children admitted to paediatric intensive care units in England and Wales: a retrospective cohort study. Lancet 2010;376:698-704

4. Ramnarayan P, Dimitriades K, Freeburn L, et al. Interhospital Transport of Critically III Children to PICUs in the United Kingdom and Republic of Ireland: Analysis of an International Dataset. Pediatr Crit Care Med 2018;19:e300-e11.

5. Paediatric Intensive Care Audit Network (PICANet). 2014 Annual Report - Tables and Figures. Universities of Leeds and Leicester: Leeds, 2015.

6. Colville G, Orr F, Gracey D. "The worst journey of our lives": parents' experiences of a specialised paediatric retrieval service. Intensive Crit Care Nurs 2003;19:103-8.

7. Colville G, Darkins J, Hesketh J, et al. The impact on parents of a child's admission to intensive care: integration of qualitative findings from a cross-sectional study. Intensive Crit Care Nurs 2009;25:72-9.

8. Suttorp MM, Siegerink B, Jager KJ, et al. Graphical presentation of confounding in directed acyclic graphs. Nephrol Dial Transplant 2015;30:1418-23.

9. Greenland S. An introduction to instrumental variables for epidemiologists. Int J Epidemiol 2000;29:722-9.

10. Horstman M, Aldiss S, Richardson A, et al. Methodological issues when using the draw and write technique with children aged 6 to 12 years. Qual Health Res 2008;18:1001-11.

11. Murphy J, Boa S. Using the WHO-ICF with talking mats to enable adults with long-term communication difficulties to participate in goal setting. Augment Altern Commun 2012;28:52-60.

12. Braun V, Clarke V. What can "thematic analysis" offer health and wellbeing researchers? Int J Qual Stud Health Well-being 2014;9:26152.

13. National Institute for Health and Care Excellence (NICE). Guide to the methods of technology appraisal, 2013.

14. Curtis LB A. Unit Costs of Health and Social Care 2015: Personal Social Services Research Unit: University of Kent, 2015.

15. Horsman J, Furlong W, Feeny D, et al. The Health Utilities Index (HUI): concepts, measurement properties and applications. Health Qual Life Outcomes 2003;1:54.

16. Varni JW, Seid M, Kurtin PS. PedsQL 4.0: reliability and validity of the Pediatric Quality of Life Inventory version 4.0 generic core scales in healthy and patient populations. Med Care 2001;39:800-12.

17. Khan KA, Petrou S, Rivero-Arias O, et al. Mapping EQ-5D utility scores from the PedsQL ${ }^{\mathrm{TM}}$ generic core scales. Pharmacoeconomics 2014;32:693-706.

18. Barber J, Thompson S. Multiple regression of cost data: use of generalised linear models. J Health Serv Res Policy 2004;9:197-204.

19. Kreif N, Grieve R, Sadique MZ. Statistical methods for costeffectiveness analyses that use observational data: a critical appraisal tool and review of current practice. Health Econ 2013;22:486-500.

20. Husereau D, Drummond M, Petrou S, et al. Consolidated Health Economic Evaluation Reporting Standards (CHEERS) statement. Int $J$ Technol Assess Health Care 2013;29:117-22.

21. Field D, Hodges S, Mason E, et al. Survival and place of treatment after premature delivery. Arch Dis Child 1991;66(4 Spec No):408-11. 